\title{
The Genetic Basis of Differences in Cation Chemoreception Sensitivity in Plasmodia of the Myxomycete Physarum polycephalum
}

\author{
By TETSUO UEDA*† AND MICHAEL J. CARLILE \\ Department of Pure and Applied Biology, Imperial College of Science and Technology, \\ London SW7 2BB, U.K.
}

(Received 18 January 1983)

\begin{abstract}
Hybridization experiments were carried out between strains of the myxomycete Physarum polycephalum which differed in their sensitivities to the cations $\mathrm{Na}^{+}, \mathrm{K}^{+}, \mathrm{Mg}^{2+}$ and $\mathrm{Ca}^{2+}$ as determined by the threshold for changes in membrane potential on application of these ions. The sensitivities were explicable on the basis of control by three genes (mon, mag and cal) for monovalent cations, magnesium and calcium, respectively, with low sensitivity dominant. Departures from the expected ratios for three unlinked loci are explicable by high sensitivity for divalent cations reducing viability, but the additional possibility that high divalent cation sensitivity cannot be expressed in the presence of low monovalent cation sensitivity has not been excluded. Chemotactic thresholds corresponded with membrane potential thresholds.
\end{abstract}

\section{INTRODUCTION}

Plasmodia of the myxomycete Physarum polycephalum show chemotaxis, moving towards higher concentrations of some substances and away from others (Ueda \& Kobatake, 1982). Chlorides cause negative chemotaxis, with the threshold for repulsion being determined by the nature of the anion (Ueda et al., 1975). The concentration threshold for the chemotactic response to salts is also the threshold for a change in membrane potential and zeta potential, so the chemoreception step in the sensory response can be studied by electrophysiological methods (Hato et al., 1976). The threshold, determined with a plasmodial strain extensively used in studies at Hokkaido University, was found to be about $1000 \mu \mathrm{M}$ for $\mathrm{NaCl}$ and $\mathrm{KCl}$ and about $100 \mu \mathrm{M}$ for $\mathrm{MgCl}_{2}$ and $\mathrm{CaCl}_{2}$ (Ueda et al., 1975). This Hokkaido strain was originally obtained from a biological supply company by Professor R. D. Allen, Dartmouth College, Hanover, N. H., U.S.A. Concentration thresholds for the four cations were subsequently determined with a range of plasmodial strains (Ueda \& Carlile, 1981). Some strains were found to have, for some cations, concentration thresholds an order of magnitude lower than for the Hokkaido strain. Four patterns of high sensitivity for the cations $\mathrm{Na}^{+}, \mathrm{K}^{+}, \mathrm{Mg}^{2+}$ and $\mathrm{Ca}^{2+}$ were observed, namely, to monovalent cations alone, to monovalent cations and $\mathrm{Mg}^{2+}$, to monovalent cations and $\mathrm{Ca}^{2+}$ and to all four cations. We here report experiments designed to elucidate the genetic basis of cation sensitivity: these involve the production of the haploid amoeboid phase by sporulation of the diploid plasmodia, and the mating of amoebae to produce hybrid plasmodia, as well as the utilization of haploid plasmodia which can arise in some amoeba clones.

\section{METHODS}

Sources of strains. The following strains, or hybrid progeny from them, were employed : a plasmodial strain from Carolina Biological Supply, designated Carolina; amoeba strains IC 121, IC 139, IC 144 and IC 149, which are progeny of the plasmodial strain a $\times i$ (Dee, 1962); amoeba strains LU 213 and LU 862 which are of hybrid origin, having a mixed Wisconsin, Indiana and Colonia background (Dee, 1982).

$\dagger$ Present address: Faculty of Pharmaceutical Sciences, Hokkaido University, Sapporo 060, Japan. 
Maintenance of strains. Amoeboid strains were cultured on Escherichia coli lawns in Petri dishes or, for storage at $5{ }^{\circ} \mathrm{C}$, on slopes in screw cap plastic vials on liver extract agar containing $p$-aminobenzoic acid, LE-PABA agar (Dee, 1966). Plasmodial strains were cultured on a semi-defined agar medium containing starch, $\mathrm{SD}(\mathrm{St})$ agar (Carlile, 1976), and stored as sclerotia. New amoeba strains were produced by allowing plasmodia to sporulate in daylight, carrying out serial dilution of spores, isolating colonies developing from single spores after plating, and then recloning the amoebae. New plasmodial strains were produced by mating amoebae. Inocula from two amoeba strains were placed close together on an E. coli lawn, and drops of semi-defined (SD) liquid medium (Carlile, 1971) and, later, sterile oatflakes added every few days to speed plasmodium growth. Plasmodia were then freed from $E$. coli by migration on LE-PABA agar and transferred to SD(St) agar.

Determination of thresholds for chemoreception. The double chamber method of Kamiya was used (Ueda et al., 1975). Plasmodia were transferred first to water agar and fed on oatflakes and finally to water agar without food. A single plasmodium was then placed in a double chamber so that it was separated electrically into two parts linked by a narrow plasmodial strand. Solutions were applied to one end of the plasmodium and the difference in membrane potential $(\Delta \psi)$ between the ends determined (Ueda et al., 1975). Below the threshold $\left(C_{\mathrm{th}}\right), \Delta \psi$ did not change appreciably, but above $C_{\mathrm{th}}$ increased linearly with the logarithm of the salt concentration $(C)$ :

$$
\Delta \psi=R \log \left(C / C_{\mathrm{th}}\right)
$$

$R$ is a constant determining slope. The values of $R$ varied from 10 to $20 \mathrm{mV}$ for different strains. Extrapolation of this dose-response plot to $\Delta \psi=0$ gives $C_{\mathrm{th}}$. Strains having for an ion a $C_{\mathrm{th}}$ of about one-tenth of that of the Hokkaido strain were recorded as being of high sensitivity for that ion. As found previously (Ueda \& Carlile, 1981), single plasmodia of some strains gave a high $C_{\mathrm{th}}$ on first testing but subsequently showed a low $C_{\mathrm{th}}$. These strains, about $10 \%$ of those tested, were recorded as being of high sensitivity.

Determination of chemotactic thresholds. Thresholds for chemotactic responses were determined by the double chamber method in which the balance pressure necessary for opposing chemotactic movement is determined (Ueda \& Kobatake, 1982) and confirmed by the double-strip method in which actual chemotactic migration is observed (Knowles \& Carlile, 1978). The two methods yielded similar results.

\section{RESULTS AND DISCUSSION}

\section{The genetic basis of sensitivity to divalent cations}

A simple genetic basis for divalent cation sensitivity is suggested by data (Ueda \& Carlile, 1981) on haploid plasmodial strains derived directly from the Colonia strain (CL) or by hybridization with Wisconsin derived strains (LU 275, LU 276, LU 278). These four plasmodial strains were all of high sensitivity to monovalent cations but differed in their sensitivity to divalent cations. One (LU 278) lacked high sensitivity to either divalent cation, one (CL) showed high sensitivity to $\mathrm{Mg}^{2+}$, one (LU 276) to $\mathrm{Ca}^{2+}$, and one (LU 285) to both divalent cations. Hence the four possible patterns of sensitivity to divalent cations can occur in haploid plasmodia; cooperation between two alleles of the same locus is not needed.

The four patterns of sensitivity could also be obtained in diploid plasmodia produced by mating amoeba LU 213 with four other amoeba strains (Table 1). These four amoeba strains must hence differ with respect to genes influencing divalent cation sensitivity, and strain LU 213 must be recessive for these genes. The same four amoeba strains mated with strain LU 862 yielded plasmodia that were of low sensitivity for $\mathrm{Mg}^{2+}$ and $\mathrm{Ca}^{2+}$ (Table 1). LU 862 must therefore be dominant for the genes influencing sensitivity, and the allele conferring low sensitivity must be dominant. The simplest hypothesis is that magnesium and calcium sensitivity are determined by separate loci (mag and $c a l$ ) with the presence of a dominant allele ( $m a g l$ or call) giving low sensitivity and the corresponding recessive allele (mag2 or cal2) giving high sensitivity in haploid plasmodia or in diploid plasmodia when homozygous.

Strains with high sensitivity to either or both divalent cations with respect to membrane potential change had correspondingly low thresholds for chemotactic repulsion by divalent cations.

\section{The control of sensitivity to monovalent cations}

All plasmodia tested were either of high or of low sensitivity to both $\mathrm{Na}^{+}$and $\mathrm{K}^{+}$. The most simple hypothesis is that monovalent cation sensitivity is determined by a single gene mon. 
Table 1. Sensitivity to divalent cations of the eight plasmodial strains produced by mating four amoeba strains with the amoeba strains LU 213 and LU 862

\begin{tabular}{|c|c|c|c|c|}
\hline \multirow[b]{3}{*}{ Amoeba strain* } & \multicolumn{4}{|c|}{ Cation sensitivity of strain: } \\
\hline & \multicolumn{2}{|c|}{ LU 213 (mag2, cal2) $\dagger$} & \multicolumn{2}{|c|}{ LU $862($ magl, call) $\dagger$} \\
\hline & $\mathrm{Mg}^{2+}$ & $\mathrm{Ca}^{2+}$ & $\mathrm{Mg}^{2+}$ & $\mathrm{Ca}^{2+}$ \\
\hline IC 121 (magl, call) & $\mathrm{L}$ & L & L & $\mathbf{L}$ \\
\hline IC 144 (magl, cal2) & $\mathrm{L}$ & $\mathrm{H}$ & $\mathrm{L}$ & $\mathrm{L}$ \\
\hline IC 149 (mag2, call) & $\mathrm{H}$ & $\mathrm{L}$ & $\mathrm{L}$ & $\mathrm{L}$ \\
\hline IC 139 (mag2, cal2) & $\mathrm{H}$ & $\mathrm{H}$ & $\mathrm{L}$ & $\mathbf{L}$ \\
\hline
\end{tabular}

L, Low sensitivity; $\mathbf{H}$, high sensitivity.

* The four amoeba strains are all progeny of the Wisconsin-derived strain a $\times i$.

+ The postulated genotype of amoeba strains is shown in parentheses: mag and cal represent loci controlling sensitivity to $\mathrm{Mg}^{2+}$ and $\mathrm{Ca}^{2+}$, respectively, with magl and call being dominant alleles giving low sensitivity and mag 2 and cal 2 recessive alleles giving high sensitivity in the absence of the corresponding dominant allele.

Experiments were carried out to examine this hypothesis and to determine whether high or low sensitivity is dominant.

The eight plasmodial strains tested for divalent cation sensitivity (Table 1) were all of high sensitivity to monovalent cations. If high sensitivity is due to the recessive condition, then all of the six amoeba strains from which they were derived must carry the recessive allele. If it is due to the dominant condition, then some at least must carry the dominant allele. The Carolina plasmodium, which is of low sensitivity to monovalent as well as divalent cations, was sporulated and amoeba clones isolated. Six plasmodial strains were obtained by mating the six amoebal strains listed in Table 1 with a Carolina amoeba strain (IC 152). All six plasmodial strains were of low sensitivity for monovalent as well as divalent cations. This result is explicable if low sensitivity for monovalent cations results from the presence of a dominant allele (monI) and high sensitivity from the presence of the corresponding recessive allele (mon 2 ) in the homozygous condition.

Matings of amoeba strains LU 213 and IC 139 with five further Carolina amoeba strains all yielded plasmodia that were of low monovalent and divalent cation sensitivity. This suggests that Carolina amoebae carry dominant genes for cation sensitivity, having the genotype monl, magl, call.

Chemotactic thresholds paralleled monovalent cation sensitivities as demonstrated by membrane potential measurements.

\section{Segration test on the three gene hypothesis of cation sensitivity}

If sensitivity to monovalent cations, $\mathrm{Mg}^{2+}$ and $\mathrm{Ca}^{2+}$ is controlled by three separate loci with dominant and recessive alleles, then it should be possible to produce plasmodial strains with eight different patterns of cation sensitivity (Table 2), the five already known (types I, V-VIII) and three others (II-IV). A plasmodial strain produced by mating IC 139 (postulated genotype mon2, mag2, cal2) and IC 152 (postulated genotype mon1, magl, call) was sporulated and 30 amoeba clones isolated. Since meiosis occurs during sporulation, eight cation sensitivity genotypes were expected. The amoeba clones were then mated with amoeba strain LU 213 to give plasmodia. LU 213 is regarded as recessive for the three genes, so the genotypes of the IC $139 \times$ IC 152 progeny amoebae should have been expressed. The cation sensitivities of the resulting plasmodia indicated that recombination between the three loci took place, but only the five sensitivity types already known were observed (Table 2). The divergence from the expectation, on the basis of three unlinked loci, of all sensitivity types occurring with equal frequency was highly significant $\left(\chi^{2}=48.4\right.$; degrees of freedom $\left.=7 ; P \ll 0.001\right)$.

Any interpretation of the divergence from the expected ratios must take into account the low frequency of the plasmodial type VIII showing high sensitivity to all cations (three plasmodia) as 
Table 2. Frequencies of different classes of cation sensitivity observed in plasmodia produced by mating the progeny of a plasmodium heterozygous for all three cation sensitivities

$\left(\right.$ IC $139 \times$ IC 152) with an amoeba strain recessive for all three sensitivities $(L U 213)^{*}$

$\begin{array}{lcccc}\begin{array}{c}\text { Plasmodial } \\ \text { type }\end{array} & \mathrm{Na}^{+}, \mathrm{K}^{+} & \mathrm{Mg}^{2+} & \mathrm{Ca}^{2+} & \begin{array}{c}\text { Observed } \\ \text { frequency }\end{array} \\ \text { I } & \mathrm{L} & \mathrm{L} & \mathrm{L} & 14 \\ \text { II } & \mathrm{L} & \mathrm{L} & \mathrm{H} & 0 \\ \text { III } & \mathrm{L} & \mathrm{H} & \mathrm{L} & 0 \\ \text { IV } & \mathrm{L} & \mathrm{H} & \mathrm{H} & 0 \\ \text { V } & \mathrm{H} & \mathrm{L} & \mathrm{L} & 9 \\ \text { VI } & \mathrm{H} & \mathrm{L} & \mathrm{H} & 2 \\ \text { VII } & \mathrm{H} & \mathrm{H} & \mathrm{L} & 2 \\ \text { VIII } & \mathrm{H} & \mathrm{H} & \mathrm{H} & 3 \\ & & & & \text { Total . . . } 30\end{array}$

Postulated plasmodial genotype

mon1, mag1, call/mon2, mag2, cal2 mon1, mag1, cal2/mon2, mag2, cal2 mon1, mag2, call/mon2, mag2, cal2 mon1, mag2, cal2/mon2, mag2, cal2 mon2, mag1, call/mon2, mag2, cal2 mon2, mag1, cal2/mon2, $\operatorname{mag} 2, \mathrm{cal} 2$ mon2, mag2, call/mon2, mag2, cal2 mon2, mag2, cal2/mon2, mag2, cal2

* The postulated genotype for IC $139 \times$ IC 152 was mon2, mag2, cal2/mon1, mag1, call and for LU 213, mon2, $\operatorname{mag} 2, \mathrm{cal} 2$.

$\dagger \mathrm{L}$, Low sensitivity; $\mathrm{H}$, high sensitivity.

$\ddagger$ On basis of a $5: 1: 1: 1: 5: 1: 1: 1$ ratio, $\chi^{2}=8 \cdot 63$, degrees of freedom $=7, P \bumpeq 0 \cdot 3$. For a $5: 0: 0: 0: 5: 1: 1: 1$ ratio, $\chi^{2}=1 \cdot 37$, degrees of freedom $=7, P>0.975$.

compared to that (type I) showing low sensitivity to all cations (14 plasmodia). This departure from the expected $1: 1$ ratio for the two parental types is significant $\left(\chi^{2}=7 \cdot 1\right.$; degrees of freedom $=1 ; P<0.01$ ). It indicates that the haploid products (spores or amoebae) of the sporulation of plasmodium IC $139 \times$ IC 152 differ in viability with amoebae of genotype mon 2 , mag 2 , cal 2 having $3 / 14$ (about $20 \%$ ) of the viability of monl, magl, call. The hypothesis that high sensitivity for any divalent cation reduces viability of amoebae to $20 \%$ would give an expected ratio of plasmodial sensitivity type of $5: 1: 1: 1: 5: 1: 1: 1$, which is in conformity with results. However, the hypothesis that high divalent cation sensitivity reduces amoeba viability to $20 \%$ in the presence of high monovalent cation sensitivity and to zero in the presence of low monovalent cation sensitivity, rendering sensitivity types II-IV unobtainable, is also tenable.

Further studies to discriminate between these possibilities were carried out, involving the attempted production of plasmodia of sensitivity types II-IV.

\section{Attempted production of plasmodia with low sensitivity to monovalent and high sensitivity to one or more divalent cations}

Five plasmodial strains (A-E) having low sensitivity to all cations and regarded as heterozygous for all three sensitivity loci were sporulated and amoeba clones isolated. These were mated to LU 213 (mon2, mag2, cal2) and the cation sensitivities of the resulting plasmodia recorded (Table 3). Data on the progeny of plasmodia A-C and D-E were analysed separately, as their genetic backgrounds differed (Table 3, see footnote). In plasmodial groups A-C, one parental type (VIII) occurred at $25 \%$ of the frequency of the other (I), a result similar to that obtained with the plasmodia analysed in Table 2 . In groups D-E, however, the two parental types did not differ in frequency. It is concluded that the effect of high divalent cation sensitivity in reducing viability of amoebae of high monovalent cation sensitivity is dependent on genetic background. Plasmodial sensitivity types II-IV were absent in all groups. Their absence both in groups $\mathrm{A}-\mathrm{C}$ and $\mathrm{D}-\mathrm{E}$ can be accounted for on the hypothesis that in the presence of low monovalent cation sensitivity, high divalent cation sensitivity reduces amoeba viability to nearly zero. An alternative possibility, however, is that in the presence of low monovalent cation sensitivity, high divalent cation sensitivity cannot be expressed. The experiments reported in Table 3 permit discrimination between these hypotheses of differential viability and expression. 
Table 3. Cation sensitivities of plasmodia in relation to hypotheses of differential viability of amoebae

\begin{tabular}{|c|c|c|c|c|c|c|c|c|c|c|}
\hline \multirow{2}{*}{$\begin{array}{l}\text { Plasmodial } \\
\text { type }\end{array}$} & \multicolumn{3}{|c|}{$\begin{array}{c}\text { Cation } \\
\text { sensitivity* }\end{array}$} & \multicolumn{7}{|c|}{$\begin{array}{l}\text { Observed frequencies among plasmodia } \\
\text { derived from plasmodia } \mathrm{A}-\mathrm{E} \dagger\end{array}$} \\
\hline & $\mathrm{Na}^{+}, \mathrm{K}^{+}$ & $\mathrm{Mg}^{2+}$ & $\mathrm{Ca}^{2+}$ & A & B & $\mathrm{C}$ & $A+B+C t$ & $\mathrm{D}$ & $\mathrm{E}$ & $\mathrm{D}+\mathrm{E} \S$ \\
\hline I & $\mathrm{L}$ & $\mathbf{L}$ & $\mathrm{L}$ & 11 & 8 & 5 & 24 & 5 & 4 & 9 \\
\hline II & $\mathrm{L}$ & $\mathbf{L}$ & $\mathrm{H}$ & 0 & 0 & 0 & 0 & 0 & 0 & 0 \\
\hline III & $\mathrm{L}$ & $\mathrm{H}$ & $\mathrm{L}$ & 0 & 0 & 0 & 0 & 0 & 0 & 0 \\
\hline IV & $\mathrm{L}$ & $\mathrm{H}$ & $\mathrm{H}$ & 0 & 0 & 0 & 0 & 0 & 0 & 0 \\
\hline V & $\mathrm{H}$ & $\mathrm{L}$ & $\mathbf{L}$ & 5 & 3 & 6 & 14 & 5 & 5 & 10 \\
\hline VI & $\mathrm{H}$ & $\mathbf{L}$ & $\mathrm{H}$ & 2 & 2 & 1 & 5 & 2 & 1 & 3 \\
\hline VII & $\mathrm{H}$ & $\mathrm{H}$ & L & 2 & 2 & 1 & 5 & 3 & 3 & 6 \\
\hline VIII & $\mathrm{H}$ & $\mathrm{H}$ & $\mathrm{H}$ & 1 & 3 & 2 & 6 & 4 & 3 & 7 \\
\hline Total & & & & 21 & 18 & 15 & 54 & 19 & 16 & 35 \\
\hline
\end{tabular}

* L, Low sensitivity; $\mathrm{H}$, high sensitivity.

$\dagger$ Five amoebae clones were selected from among the 30 obtained (Table 2) by sporulating the Wisconsin $(a \times$ i)-Carolina hybrid IC $139 \times$ IC 152. Three were mated to the predominantly Colonia strain LU 213 to give plasmodia A-C and two backcrossed to IC 139 to give plasmodia D and E. Plasmodia A-E, which were of low sensitivity to all three cations and of presumed genotype mon 1, magl, call/mon2, mag2, cal2 were sporulated and the 89 amoeba clones isolated from them mated to LU 213 (mon2, mag2, cal2).

¥Comparison with expected frequencies on various hypotheses. Equal frequency of all plasmodial types, $\chi^{2}=73 \cdot 1, P \ll 0.001$. Equal frequency of types I and VIII, $\chi^{2}=10.8, P \bumpeq 0.001$. Ratio $4: 1: 1: 1: 4: 1: 1: 1$, $\chi^{2}=46 \cdot 1, P \ll 0 \cdot 001$. Ratio $4: 0: 0: 0: 4: 1: 1: 1, \chi^{2}=2 \cdot 23, P \bumpeq 0.95$.

$\S$ Comparison with expected frequencies on various hypotheses. Equal frequency of all plasmodial types $\chi^{2}=23 \cdot 9, P \ll 0 \cdot 001$. Equal frequency of types I and VIII, $\chi^{2}=0 \cdot 25, P \bumpeq 0 \cdot 6$. Ratio $1: 0: 0: 0: 1: 1: 1: 1$, $\chi^{2}=4.29, P \bumpeq 0.75$.

\section{Examination of the viability and expression hypotheses for the absence of high divalent cation sensitivity in the presence of low monovalent cation sensitivity}

Plasmodia A-E will, if the viability hypothesis is correct, all have the same cation sensitivity genotype, mon 1, mag1, call/mon 2, mag2, cal2. On sporulation they should all yield the five viable amoeba genotypes and these on mating with mon2, mag2, cal2 amoebae should give the five known plasmodial sensitivity types. On the expression hypothesis, however, plasmodia A-E can have four possible genotypes, those listed as I-IV in Table 3. On sporulation, plasmodial genotype I should yield eight different amoeba genotypes and these on mating with mon2, mag2, cal 2 amoebae should give the same five plasmodial sensitivity types as are expected on the viability hypothesis. Plasmodial genotypes II-IV, however, will yield fewer amoeba genotypes and fewer plasmodial sensitivity types (Table 4). Hence should any of the plasmodia A-E yield less than five plasmodial sensitivity types among the progeny, the expression hypothesis is proved. In fact, however, all five plasmodia yielded five sensitivity types among their progeny (Table 3) indicating that plasmodia A-E all have genotype I. This would be inevitable on the viability hypothesis.

On the expression hypothesis, if the four possible amoeba genotypes that carry monl do not differ in viability, then there is a probability of 0.25 that any one of the plasmodia A-E will be of genotype I and a probability of $(0 \cdot 25)^{5}=<0.001$ that all will be. However one set of the amoebae used in constructing plasmodia A-E were progeny of IC $139 \times$ IC 152; here mag 2 or cal 2 in the presence of mon 2 reduces amoeba viability to $20 \%$, and it is prudent to assume that this might also occur in the presence of monl. If this is so, the relative probabilities of any one of the plasmodia A-E belonging to genotypes I-IV is $5: 1: 1: 1$ and to genotype I, $5 / 8=0.625$. So the probability of all five being of genotype I is $(0.625)^{5} \bumpeq 0.095$. The expression hypothesis as an explanation for the absence of sensitivity types II-IV is hence improbable but cannot be excluded. 
Table 4. Expected occurrence, on the basis of the expression hypothesis, of plasmodial sensitivity genotypes and phenotypes among the progeny of plasmodia of low sensitivity for both monovalent and divalent cations

The cation sensitivity genotypes and phenotypes are represented by Roman numerals, see Table $2 .+$ and 0 indicate respectively the presence or absence of a given genotype. The phenotype frequency is given in parentheses.

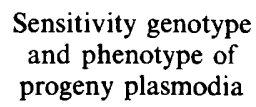

modia

$\begin{array}{lllll}\text { I } & +(3) & 0(2) & 0(2) & 0(1) \\ \text { II } & +(0) & +(0) & 0(0) & 0(0) \\ \text { III } & +(0) & 0(0) & +(0) & 0(0) \\ \text { IV } & +(0) & +(0) & +(0) & +(0) \\ \text { V } & +(1) & 0(0) & 0(0) & 0(0) \\ \text { VI } & +(1) & +(1) & 0(0) & 0(0) \\ \text { VII } & +(1) & 0(0) & +(1) & 0(0) \\ \text { VIII } & +(1) & +(1) & +(1) & +(1)\end{array}$

Differential viability of amoeba genotypes has to be invoked to account for the low frequency of one parental type among the progeny of some matings. The most simple hypothesis to account for the ratios obtained is hence that both the genes mag2 and cal 2 may depress viability in the presence of mon 2 but reduce viability to very low levels, probably zero, in the presence of monl. It is not necessary or helpful to invoke linkage as an explanation for the ratios obtained.

We wish to thank the British Council for a studentship to one of us (T.U.), which made this study possible, Professor R. D. Allen, Dr Jennifer Dee and Dr Alan Wheals for providing strains, and Dr Jennifer Dee and Dr Bernard Lamb for commenting upon the manuscript and helpful discussion.

\section{REFERENCES}

CARLILE, M. J. (1971). Myxomycetes and other slime moulds. Methods in Microbiology 4, 237265.

Carlile, M. J. (1976). The genetic basis of the incompatibility reaction following plasmodial fusion between different strains of the myxomycete $P h y$ sarum polycephalum. Journal of General Microbiology 93, 371-376.

DEE, J. (1962). Recombination in a myxomycete, Physarum polycephalum. Genetical Research 3, 11-23.

DEE, J. (1966). Multiple alleles and other factors affecting plasmodium formation in the true slime mould Physarum polycephalum. Journal of Protozoology 13, 610-616.

DEE, J. (1982). Genetics of Physarum polycephalum. In Cell Biology of Physarum and Didymium vol. I, pp. 211-251. Edited by H. C. Aldrich \& J. W. Daniel. New York: Academic Press.

Hato, M., Ueda, T., Kurihara, K. \& Kobatake, K. (1976). Change in zeta potential and membrane potential of slime mold Physarum polycephalum in response to chemical stimuli. Biochimica et biophysica acta 426, 73-80.

Knowles, D. J. C. \& Carlile, M. J. (1978). The chemotactic response of plasmodia of the myxomycete Physarum polycephalum to sugars and related compounds. Journal of General Microbiology 108, 17 25.

UEDA, T. \& CARLILE, M. J. (1981). Strain differences in sensitivity of cation chemoreception in Physarum polycephalum. In Biology of Physarum, pp. 246-250. Edited by L. Rakoczy. Cracow: Jagiellonian University Press

Ueda, T. \& Kobatake, Y. (1982). Chemotaxis in plasmodia of Physarum polycephalum. In Cell Biology of Physarum and Didymium, vol. I, pp. 111-143. Edited by H. C. Aldrich \& J. W. Daniel. New York: Academic Press.

Ueda, T. Terayama, K., Kurihara, K. \& Kobatake, K. (1975). Threshold phenomena in chemoreception and taxis in the slime mould Physarum polycephalum. Journal of General Physiology 65, 223-234. 